\title{
Performance Analysis of On-line Batch Optimization Systems
}

\author{
C. Loeblein* .J.D. Perkins*, B. Srinivasan ${ }^{\dagger}$ and D. Bonvin ${ }^{\dagger}$ \\ * Centre for Process Systems Engineering \\ Imperial College \\ London SII $2 \mathrm{BI}$. LK \\ Email: \{c.locblein, j.perkins\} ops.ir.ac.uk \\ ${ }^{\dagger}$ Institut d.Automatique \\ Ecole Polvtechnique Federale de Lausanne \\ $\mathrm{CH}-1015$ Lausanne, Switzerland \\ Email: \{srinivasan, bonvin\}@ia.epfl.ch
}

\begin{abstract}
In this paper, the on-line optimization of batch reactors under parametric uncertainty is considered. A method is presented that estimates the likely economic performance of the on-line optimizer. The method of orthogonal collocation is mployed to convert the differential algebraic optimization problem (DAOP) of the dynamic optimization into a nonlinear program (NLP) and determine the nominal optimum. Based on the resulting NLP, the optimization steps are approximated by neighbouring extremal problems and the average deviation from the true process optimum is determined dependent on the measurement error and the parametric uncertainty. A back off from the active path and endpoint constraints is determined at each optimization step which ensures the feasible operation of the process.

The method of the average deviation from optimum is developed for time optimal problems. The theory is demonstrated on an example.
\end{abstract}

\section{INTRODUCTION}

A wide variety of products in the chemical industries are produced in batch mode. Due to disturbances during operation and uncertainties in process parameters, such as reaction kinetic parameters, there is a danger of producing unsatisfactory batches where the product or safety specifications are not met and path or endpoint constraints are violated. Therefore, it is desirable to supervise and optimize the process during its operation in order to meet the product and safety specifications while maximizing an objective function, for example the yield of the desired product. This can be achieved by acquiring on-line process information which is then used to determine an improved operation policy for the rest of the batch. This results in the following on-line optimization scheme which consists of two steps, as depicted in Figure 1. In a first step, the process model is identified or updated by estimating the state variables and/or a set of

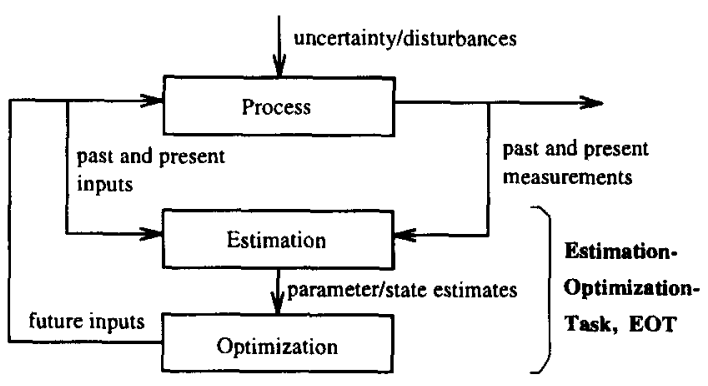

Figure 1: General structure of an on-line batch optimization system. parameters using past and present process measurements. The updated model is then optimized with respect to the manipulated variables and a new optimal input trajectory over the remaining time horizon is determined. This sequence of an estimation and optimization step is referred to in the following as an Estimation-Optimization-Task, EOT (Ruppen et al., 1997). The first part of the calculated input trajectory is applied to the process until a new EOT is carried out at some future point in time.

\section{ORTHOGONAL COLLOCATION}

Since in batch processes the dynamic behaviour is dominating and usually no steady state is reached the objective function needs to be optimized with respect to the dynamic model equations. Time optimal problems, where the only objective is the minimization of the final batch time, have the following form:

$$
\begin{array}{ll}
\min _{u, t_{f}} & t_{f} \\
\text { s.t. } & \dot{x}=f(x, u, p, t), \quad x\left(t_{0}\right)=x_{0} \\
& g(x, u, p, t) \leq 0,
\end{array}
$$

with $x$ representing the states, $u$ the control inputs and $p$ the uncertain process parameters. Since besides the dynamic model equations, $f$ a set of algebrai path/endpoint constraints, $g$ is present, this problem is called a differential algebraic optimization problem, DAOP. One or more conditions, which are represented by a subset of the constraints $g$, need to be reached in the minimum possible time. This subset of the constraints corresponds to the so-called 
terminal conditions, which define the moment when the terminal time $t_{f}$ is reached.

One method that circumvents the numerically expensive integration of the system and allows the easy incorporation of algebraic path/endpoint constraints and discontinuities in the inputs is the method of orthogonal collocation (Cuthrell and Biegler, 1987). In this method, the system is solved and optimized simultaneously. This is achieved by converting the DAOP into a nonlinear algebraic optimization problem, NLP. The conversion into an NLP consists of two steps: parametrization and discretization. In the first step, the input and state variable profiles, $u$ and $x$ are approximated by polynomials parametrized by $\xi$ and $\nu$. In the second step, the dynamic model equations are discretized and the residual equations are enforced on a finite number of collocation points in order to obtain a finite dimensional problem. Together with the algebraic path and endpoint constraints, the DAOP (1)-(3) is converted into the following NLP in $\xi, \nu$ and $t_{f}$ which can be solved with standard NLP solvers:

$$
\begin{aligned}
\min _{\xi, \nu, t_{f}} & t_{f} \\
\text { s.t. } & \dot{X}\left(\xi, \nu, t_{i}\right)-f\left(\xi, \nu, p, t_{i}\right)=0 \\
& g\left(\xi, \nu, p, t_{i}\right) \leq 0, \quad \forall t_{i} .
\end{aligned}
$$

In this approach, the dynamic model equations are considered as algebraic equality constraints which only need to be satisfied at the final solution, but not at every iteration during the optimization. This is also referred to as an infeasible path method.

Usually the input and state variable profiles are approximated by piecewise polynomials on a number of finite elements. This improves the approximation of sharply changing profiles, where a global approximation would require a very high degree of the approximation polynomial. Furthermore, superelements are introduced which allow the definition of discontinuities in the input variables, see Figure 2. In this case,

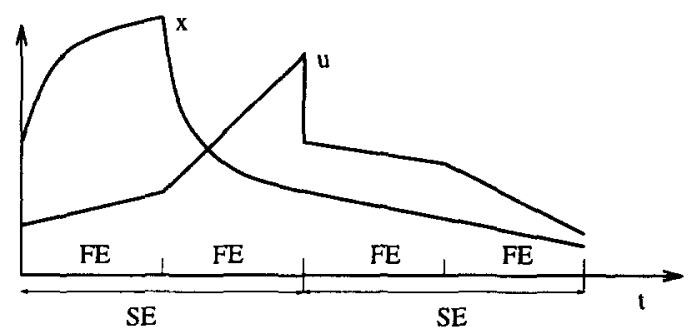

Figure 2: Piecewise approximation of input and state profiles on finite elements and superelements.

the continuity conditions for the input and state variable profiles at the boundaries of the finite elements and for the state variable profiles at the boundaries of the superelements are added to the equations of the NLP (4)-(6). A detailed description of the method of orthogonal collocation can be found in the paper by Cuthrell and Biegler, (1987).

\section{AVERAGE DEVIATION FROM OPTIMUM}

Due to the different error sources which are present during on-line optimization, such as measurement errors and parametric uncertainties, the optimizer will usually not predict the true optimum, but there will be a deviation from the same. Therefore, the performance of an on-line batch optimization system depends on the available measurements together with their quality and the amount of uncertainty in the process parameters. For continuous processes, the method of the average deviation from optimum (de Hennin et al., 1994; Loeblein and Perkins, 1996) was developed in order to estimate the likely economic performance of a given structure of an on-line optimization system. In this paper, the method of the average deviation from optimum is extended to the time optimal operation of batch reactors under uncertainty. It estimates the economic performance of an on-line optimization system by analysing how close to the true optimum it is possible to operate the process. This performance can be compared against off-line optimization and the economic benefit of on-line optimization identified. Also, the relative performance of different on-line optimization systems, involving for example different choices of measured and manipulated variables or different estimated parameters, may be compared. The error sources are described by

- a normally distributed measurement error, $\epsilon$ with given standard deviation $\sigma_{\epsilon}$ and

- a normally distributed parameter uncertainty around a nominal value, $\eta$ with given standard deviation, $\sigma_{\eta}$.

In the analysis which follows, it will be assumed that the optimization using orthogonal collocation is carried out with respect to piecewise constant, equally distributed input variables, see Figure 3. This implies that the inputs are approximated by a zero order polynomial on equally distributed superelements with one finite element defined on each superelement. The state variable profiles are approximated by first or higher order polynomials in order to give a good approximation of the system. Furthermore, the different EOTs are carried out at the discontinuities in the input variables.

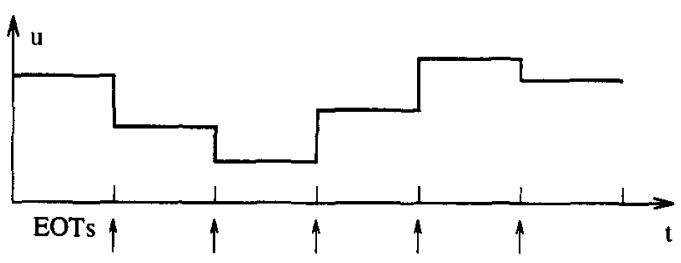

Figure 3: Input profile and EOTs.

First and second order approximation

In a first step towards the analysis, the problem (4)(6) is solved with the nominal parameter values in order to obtain the nominal optimum. Since the 
method is based on an approximation of the nonlinear problem, a first and second order perturbation model around the nominal trajectory is derived from the collocated system. Due to this representation of the problem, both the estimation and the optimization steps can be solved analytically and the effect of the error sources can be mapped through the estimation and optimization steps in order to analyse their effect on the optimizer performance. The required perturbation model is obtained from a second order Taylor series expansion of the objective function, $t_{f}$ with respect to the input variables and the uncertain process parameters. However, the input variables $u$ do not directly affect the terminal time, $t_{f}$. The terminal time is only affected by changes in the terminal conditions, i.e. the appropriate subset of the constraints $g$, which are in turn dependent on the input variables, $u$. Therefore, the second order Taylor series expansion of the objective function can only be obtained through the terminal constraints. Initially, the entire set of path and endpoint constraints which are active at the nominal optimum is linearized with respect to the piecewise constant inputs, $u$, the uncertain parameters, $p$ and the final batch time, $t_{f}$ :

$$
\delta g=\left[\begin{array}{ll}
H & H_{t_{f}}
\end{array}\right]\left[\begin{array}{c}
\delta u \\
\delta t_{f}
\end{array}\right]+G \delta p=0
$$

During the calculation of $H_{t_{f}}$, it needs to be taken into account that with changing $t_{f}$ the locations of the discontinuities in the input variables, $t_{i}$ change, since the piecewise constant inputs were assumed to be equally distributed, see Figure 4 .

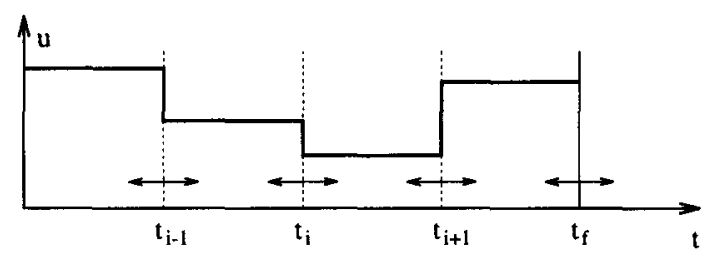

Figure 4: Change of the switching times in the inputs with changing final time.

In the case of more than one active constraint, the vector of the piecewise constant inputs, $\delta u$ and the matrix $H$ need to be partitioned according to the dimension of $\delta g$ such that $\left[H_{2} H_{t_{f}}\right]$ is a square matrix and Equation (7) is rewritten as:

$$
\left[\begin{array}{l}
\delta u_{2} \\
\delta t_{f}
\end{array}\right]=-\left[H_{2} H_{t_{f}}\right]^{-1}\left(H_{1} \delta u_{1}+G \delta p\right) .
$$

Assuming that $\left[\mathrm{HH}_{t_{f}}\right]$ is of full row rank there always exists a partition such that $\left[H_{2} H_{t_{j}}\right]$ is nonsingular. If [ $\left.H H_{t_{f}}\right]$ is not of full row rank then two or more of the constraints are linearly dependent and are affected by the inputs in the same way. The corresponding singular rows can be removed until $\left[H H_{t}\right.$ ] has full row rank. The second order sensitivities are then determined by perturbing $\delta u_{1}$ and $\delta p$ and calculating the finite differences of the perturbed first order quantities. Taking the last row of the sensitivities gives the following second order approximation of the objective function:

$$
\begin{aligned}
\delta t_{f}= & C_{1} \delta u_{1}+\delta p^{T} C_{2} \delta u_{1}+\frac{1}{2} \delta u_{1}^{T} C_{3} \delta u_{1} \\
& +C_{4} \delta p+\frac{1}{2} \delta p^{T} C_{5} \delta p
\end{aligned}
$$

Note that the minimization of $\delta t_{f}$ represents an unconstrained QP in the reduced space of the remaining degrees of freedom $\delta u_{1}$ after the active inequality constraints including terminal conditions are met. The process variables which are measured for the parameter estimation at each EOT are linearized with respect to the uncertain parameters:

$$
\delta y=J \delta p
$$

\section{Least squares parameter estimation}

The following minimization problem is solved in order to obtain estimates of the uncertain process parameters:

$$
\begin{array}{ll}
\min _{\delta \hat{p}_{i}} & \left(\delta y_{i}-\delta \hat{y}_{i}\right)^{T} Q^{T} Q\left(\delta y_{i}-\delta \hat{y}_{i}\right) \\
& +\left(\delta \hat{p}_{i}-\delta \hat{p}_{0}\right)^{T} W^{T} W\left(\delta \hat{p}_{i}-\delta \hat{p}_{0}\right) \\
\text { s.t. } & \delta \hat{y}_{i}=J_{i} \delta \hat{p}_{i} ; \quad \delta y_{i}=J_{i} \delta p+\epsilon_{i}
\end{array}
$$

where $\delta \hat{y}_{i}$ are the past model outputs and $\delta y_{i}$ is the vector of all the measurements collected in the past with normally distributed measurement errors $\epsilon_{i}$. The objective function is weighted with the covariances of the a-priori parameter uncertainty and the measurement error, $W=\operatorname{diag}\left(\sigma_{\eta}^{-1}\right)$ and $Q=$ $\operatorname{diag}\left(\sigma_{\epsilon_{i}}^{-1}\right) . \quad \delta \hat{p}_{0}$ is the a-priori estimate of the uncertain parameters. By considering the vector of all past measurements this formulation allows the calculation of an analytical expression for the parameter estimates, dependent on the measurement error and the a-priori parameter uncertainty. Due to the incorporation of a-priori knowledge of the estimated parameters, the covariance of the parameter estimate can never be bigger than the covariance of the a-priori uncertainty, regardless of the quality of the measurements.

The estimation problem can be equivalently reformulated in a recursive manner (Ljung, 1987). This is necessary in an on-line implementation of the algorithm where it is not desired to store all past measurements but update the current parameter estimate with every new measurement coming in.

\section{Optimization and back offs from active constraints}

Since it is not always possible to obtain full information about the state of a system, it is very difficult to decide when the terminal conditions of the batch are reached and the batch can be stopped. Additionally, measurement errors represent an error source even with all the states measured. Therefore, some conservatism is introduced into the optimization at each 
EOT in form of a back off from the active constraints which tries to ensure that the batch reaction is not stopped before the endpoint specifications are met. The basic idea of the back off is shown in Figure 5 . The true process optimum often lies on a boundary of the feasible region defined by one or more active path or endpoint constraints. Due to the uncertainty in the parameters and the measurement errors, it is unlikely that the optimization will predict the true optimal input variables which would operate the process exactly on this set of active constraints. Dependent on the error sources the suboptimal input variables might cause a violation of the process constraints once they are applied to the process. The back off tries to accommodate all the possible error sources and keep the variation of the process constraints due to uncertainty and measurement errors inside the feasible region of the process, while still operating as closely to the constraints as possible, Figure 5.

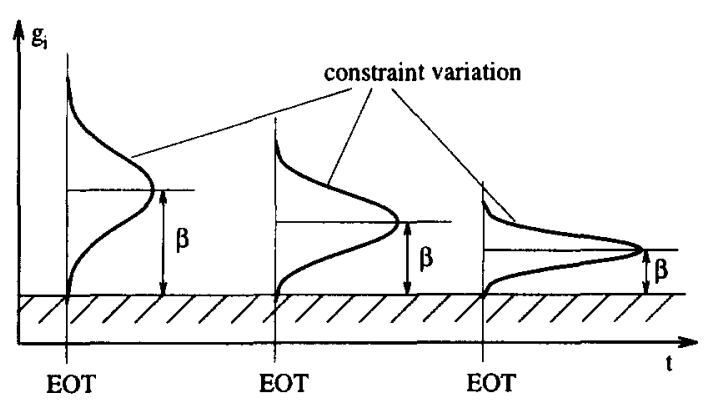

Figure 5: Back off from active constraints.

The back off, $\beta$ is introduced into the linearized equation of the constraints, Equation (7):

$$
\delta g=\left[\begin{array}{ll}
H & H_{t_{f}}
\end{array}\right]\left[\begin{array}{c}
\delta u \\
\delta t_{f}
\end{array}\right]+G \delta p+\beta=0
$$

Similar to the approach that was taken above, the second order sensitivities are determined and the following second order perturbation model of the problem including back off from the active constraints is obtained:

$$
\begin{aligned}
\delta t_{f}= & C_{1} \delta u_{1}+\delta p^{T} C_{2} \delta u_{1}+\frac{1}{2} \delta u_{1}^{T} C_{3} \delta u_{1}+C_{4} \delta p \\
& +\frac{1}{2} \delta p^{T} C_{5} \delta p+C_{6} \beta+\beta^{T} C_{7} \delta u_{1} \\
& +\beta^{T} C_{8} \delta p+\frac{1}{2} \beta^{T} C_{9} \beta
\end{aligned}
$$

At each EOT, the process is reoptimized and the optimal inputs are determined over the remaining time horizon. Taking the appropriate submatrices and neglecting the constant terms, the optimization step at each EOT is approximated for the analysis by the solution of the QP:

$$
\begin{array}{cc}
\min _{\delta u_{i 1}} & C_{i 1} \delta u_{i 1}+\delta \hat{p}_{i}^{T} C_{i 2} \delta u_{i 1}+\delta \bar{u}_{i 1} \vec{C}_{i 2} \delta u_{i 1} \\
& +\frac{1}{2} \delta u_{i 1}^{T} C_{i 3} \delta u_{i 1}+\beta_{i}^{T} C_{i 7} \delta u_{i 1} .
\end{array}
$$

This represents an unconstrained QP where the necessary back off from the process constraints appears in the objective function. It can be solved analytically dependent on the current parameter estimates, the back off from the active constraints and the inputs that were applied to the process in the past, $\delta u_{i 1}^{*}=\delta u_{i 1}^{*}\left(\beta_{i}, \delta \hat{p}_{i}, \delta \bar{u}_{i 1}\right)$. Note that only the first element of $\delta u_{i 1}^{*}$ is applied to the process since at the next discontinuity in the inputs a further EOT is carried out. The optimal input variables that are determined during on-line optimization with $n$ EOTs are a function of the set of back offs, $\beta$ and the set of parameter estimates, $\delta \hat{p}$ :

$$
\delta u_{1}^{*}\left(\beta_{0}, \ldots, \beta_{n}, \delta \hat{p}_{0}, \ldots, \delta \hat{p}_{n}\right)=\delta u_{1}^{*}(\beta, \delta \hat{p}) .
$$

The index starts at $i=0$ to allow for the back off in the first time interval, before the first EOT is carried out. The corresponding optimal values for $\delta u_{2}^{*}$ can be obtained from Equation (12).

The necessary back off from the active constraints is recomputed at every EOT and decreases the more confidence in the uncertain parameters is gained. It is determined by examining the variation of the process constraints when the inputs calculated during the optimization are applied to the process and depends on the confidence in the uncertain parameters:

$$
G_{i}\left(\delta p-\delta \hat{p}_{i}\right) \stackrel{!}{\leq} \beta_{i}
$$

Since the error sources are normally distributed and all the dependencies are linear, the variation in the active process constraint functions is also normally distributed. The variance of the process constraints can be determined dependent on the standard deviations of the parametric uncertainty and the measurement error and the back off is calculated to ensure feasible operation with a probability of $\alpha \%$.

If at a particular EOT there are more active path and/or endpoint constraints than there are degrees of freedom for optimization, the process cannot be reoptimized. Instead, it needs to be run in open loop until enough degrees of freedom are available again or the end of the batch is reached.

\section{Integration of the deviation from optimum}

Similar to the calculation of the inputs during the online optimization, the true optimum input variables can be determined by minimizing Equation (9) dependent on the normally distributed parametric uncertainty, $\delta p=\eta$. The true minimum final batch time is then obtained from Equation (9) with the true optimum input variables, $\delta u_{1}^{*}(\delta p)$ introduced. The final batch time that is achieved in reality is given by Equation (13) with the predicted optimal inputs $\delta u_{1}^{*}(\beta, \delta \hat{p})$ and the back off at the last EOT $n, \beta_{n}$ introduced. The deviation of the achieved final batch time from the true minimum final time is integrated with respect to the distribution functions of the parametric uncertainty and the measurement error and gives the average deviation from optimum 
for the on-line optimization with $n$ EOTs:

$$
\begin{aligned}
\Theta & =\int_{-\infty}^{\infty} \ldots \int_{-\infty}^{\infty}\left[\delta t_{f}\left(\delta u_{1}^{*}(\delta p), \delta p\right)\right. \\
& \left.-\delta t_{f}\left(\delta u_{1}^{*}(\beta, \delta \hat{p}), \delta p, \beta_{n}\right)\right] f(\eta) f(\epsilon) d \epsilon d \eta(17) \\
& =\Theta\left(\beta, \sigma_{\eta}, \sigma_{\epsilon}, n\right)
\end{aligned}
$$

\section{EXAMPLE}

The method of the average deviation from optimum is demonstrated on a semi batch reactor which is operated to produce 2-acetoacetyle pyrrole from pyrrole and diketene in the minimum possible time (Ruppen et al., 1997). The model of the reaction system that proved to be adequate for the case of continuous feed addition comprises the following reactions:

$$
\begin{array}{rll}
P+D & \stackrel{K}{\longrightarrow} & P A A \\
D+D & \stackrel{K}{\longrightarrow} & D H A \\
D & \stackrel{K}{\longrightarrow} & \text { oligomers } \\
P A A+D & \stackrel{K}{\longrightarrow} & F
\end{array}
$$

with $P$ : pyrrole, $D$ : diketene, $K$ : pyridine (catalyst), $P A A$ : 2-acetoacetyl pyrrole, $D H A$ : dehydroacetic acid and $F$ : by-product. Assuming constant density of the components and isothermal operation results in the following reaction model. The dilution of catalyst is considered by normalizing the rate constants with respect to the reaction volume. This is not implemented for the rate constant $k_{O}$, since the rate of oligomerization is also promoted by other intermediate products (Ruppen et al., 1997):

$$
\begin{aligned}
\frac{d c_{D}}{d t}= & -\frac{k_{A}}{v_{R}} c_{P} c_{D}-2 \frac{k_{D}}{v_{R}} c_{D}^{2}-k_{O} c_{D} \\
& -\frac{k_{F}}{v_{R}} c_{P A A} c_{D}+\frac{f}{v_{R}}\left(c_{D f}-c_{D}\right) \\
\frac{d c_{P}}{d t}= & -\frac{k_{A}}{v_{R}} c_{P} c_{D}-\frac{f}{v_{R}} c_{P} \\
\frac{d c_{P A A}}{d t}= & \frac{k_{A}}{v_{R}} c_{P} c_{D}-\frac{k_{F}}{v_{R}} c_{P A A} c_{D}-\frac{f}{v_{R}} c_{P A A} \\
\frac{d c_{D H A}}{d t}= & \frac{k_{D}}{v_{R}} c_{D}^{2}-\frac{f}{v_{R}} c_{D H A} \\
\frac{d v_{R}}{d t}= & f
\end{aligned}
$$

The concentration of diketene $D$ in the feed stream is represented by $c_{D f}$. The manipulated input variable is the feed rate, $f[l / m i n]$ of diluted diketene. The nominal values of the kinetic parameters and the initial conditions are given in Table 1.

With the necessary endtime specifications and a path constraint on the feed rate, the optimization problem can be written as follows:

$$
\begin{array}{ll}
\min _{f, t_{f}} & t_{f} \\
\text { s.t. } & \text { dynamic model equations }(19)-(23) \\
& c_{P A A}\left(t_{f}\right) v_{R}\left(t_{f}\right) \geq 0.42 \mathrm{~mol} \\
& c_{D H A}\left(t_{f}\right) \leq 0.15 \mathrm{~mol} / \mathrm{l}
\end{array}
$$

\begin{tabular}{||c|c|||c|c||}
\hline \multicolumn{2}{||c||}{ Parameters } & \multicolumn{2}{c||}{ Initial conditions } \\
\hline \hline$k_{A}$ & $0.053 l /($ mol min $)$ \\
\hline$k_{D}$ & $0.128 l /($ mol min $)$ & $c_{D 0}$ & $0.09 \mathrm{~mol} / l$ \\
\hline$k_{O}$ & $0.0281 / \mathrm{min}$ & $c_{P 0}$ & $0.72 \mathrm{~mol} / \mathrm{l}$ \\
\hline$k_{F}$ & $0.003 l /(\mathrm{mol} \mathrm{min})$ & $c_{P A A 0}$ & $0.1 \mathrm{~mol} / \mathrm{l}$ \\
\hline$c_{D f}$ & $5.82 \mathrm{~mol} / \mathrm{l}$ & $c_{D H A 0}$ & $0.02 \mathrm{~mol} / \mathrm{l}$ \\
\hline$v_{R 0}$ & $1.0 \mathrm{l}$ \\
\hline
\end{tabular}

Table 1: Nominal model parameter values and initial conditions.

$$
\begin{aligned}
& c_{D}\left(t_{f}\right) \leq 0.025 \mathrm{~mol} / \mathrm{l} \\
& f(t) \geq 0 .
\end{aligned}
$$

A detailed description of the diketene chemistry, the modelling of the reactor and the experimental set up of on-line optimization can be found in the paper by Ruppen et al., (1997).

\section{Nominal optimum}

The first step towards the analysis is the determination of the nominal optimum. The dynamic model equations in DAOP (24) are collocated on eight equally distributed superelements with one finite element per superelement. The state variables are approximated with quadratic polynomials while the in put variables are specified as piecewise constant. The resulting NLP is then solved using the nominal parameter values and initial conditions given in Table 1 . The input and state variable profiles at the nomi-

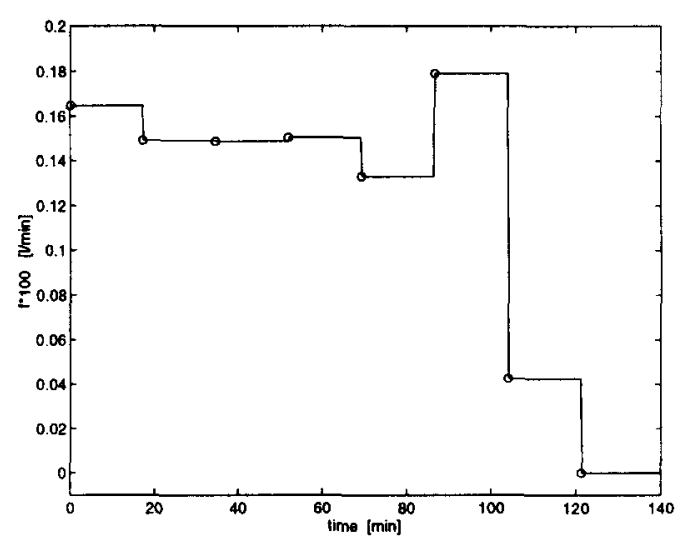

Figure 6: Input profile at nominal optimum.

nal optimum are shown in Figure 6 and 7 respectively. Due to the high number of superelements, the approximation of the system is quite accurate, as can be seen in Figure 7, where both the approximated and integrated state variables profiles are plotted. The terminal time at the nominal optimum is $t_{f}=138.62 \mathrm{~min}$. Besides the three endpoint constraints, the lower bound on the feed rate becomes active in the last superelement.

\section{Optimization analysis}

In order to demonstrate the analysis of the on-line optimization, it is assumed that the uncertain process parameters are the two rate constants $k_{A}$ and 

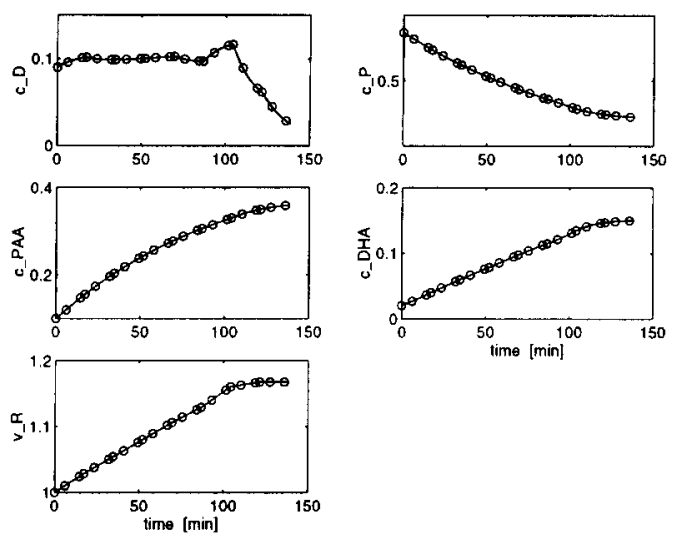

Figure 7: State variable profiles at nominal optimum.

$k_{D}$. The uncertainty is described as normally distributed with the standard deviations $\sigma_{\eta, k_{A}}=0.003$ and $\sigma_{\eta, k_{D}}=0.007$. In the following, three different structures of the on-line optimizer are analysed and their performance is compared against off-line optimization. The different structures are characterized by the selection of the measurement, $y$ which is used to update the estimates of the uncertain parameters. For that purpose, it is assumed that one or more state variables can be measured on-line. The alternatives consist of measuring either one of the concentrations $c_{P}, c_{D}$ or $c_{P A A}$. The standard deviation of the measurement error is assumed to be $1 \%$ of the approximate average nominal value of the corresponding state variable. Since the three terminal constraints and the lower bound on the feed rate in the last superelement are active at the nominal optimum, the process has to be run in open loop with respect to the last four inputs. This is necessary for the optimization to have enough degrees of freedom at the last EOT to back off from the four active constraints. The system is collocated on eight superelements which implies that four EOTs are carried out during on-line optimization.

The analysis results are shown in Table 2 . Off-line

\begin{tabular}{||c|c|c|c||}
\hline Optimization & $y$ & $\sigma_{\epsilon}$ & $\Theta$ \\
\hline \hline Off-line & - & - & $-47.56 \mathrm{~min}$ \\
\hline On-line & $c_{D}$ & 0.001 & $-39.12 \mathrm{~min}$ \\
\hline On-line & $c_{P}$ & 0.005 & $-23.44 \mathrm{~min}$ \\
\hline On-line & $c_{P A A}$ & 0.003 & $-17.86 \mathrm{~min}$ \\
\hline
\end{tabular}

Table 2: Analysis results.

optimization shows an average deviation from optimum of $\Theta=-47.56 \mathrm{~min}$. Implementing an on-line optimizer, where the diketene concentration, $c_{D}$ is measured, does not improve this result significantly. In this case, the average deviation from optimum is $\Theta=-39.12 \mathrm{~min}$. However, a much better operation of the reactor can be obtained when either the pyrrole concentration, $c_{P}$ or the acetoacetyle pyrrole concentration, $c_{P A A}$ is measured. Both options show a substantial improvement against the off-line optimiza- tion result, where measuring $c_{P A A}$ gives the least average deviation from optimum with $\Theta=-17.86 \mathrm{~min}$ compared to $\Theta=-23.44$ min when $c_{P}$ is measured.

\section{CONCLUSIONS}

The method of the average deviation from optimum allows the estimation of the economic performance of a given on-line batch optimization system. This performance can be compared against off-line optimization and the economic benefit of on-line optimization identified. Different on-line optimizer structures can be compared and the structure with the best performance can be chosen for implementation. Furthermore, the analysis method returns the necessary back off from the active path and endpoint constraints at each Estimation-Optimization-Task EOT in order to ensure the feasible operation of the process.

The method of the average deviation from optimum has been developed for time optimal problems and demonstrated on an example.

\section{NOTATION}

$\delta u^{*} \quad$ optimal piecewise constant input variables

$\delta u_{1} \quad$ degrees of freedom in the reduced optimization space

$\delta u_{2} \quad$ inputs determined by the constraints

$\delta u_{i 1} \quad$ remaining degrees of freedom in the future at $\operatorname{EOT} i$

$\delta \bar{u}_{i 1} \quad$ input variables applied to the process in the past at EOT $i$

$\delta p \quad$ uncertain process parameters

$\delta \hat{p} \quad$ parameter estimates

$\beta_{i} \quad$ back off at EOT $i$

$\eta \quad$ normally distributed parameter uncertainty

$\epsilon$ normally distributed measurement error

$\sigma \quad$ standard deviation

$\Theta$ average deviation from optimum

\section{REFERENCES}

J.E. Cuthrell and L.T. Biegler, (1987). On the optimization of differential-algebraic process systems. AIChE Journal, 33(8), 1257-1270.

S.R. de Hennin, J.D. Perkins and G.W. Barton, (1994). Structural decisions in on-line optimization. In Proc. of Int. Conf. on Process Systems Engineering PSE '94, pp 297-302.

L. Ljung, (1987). System Identification-Theory for the User. Prentice-Hall.

C. Loeblein and J.D. Perkins, (1996). Economic analysis of different structures of on-line process optimization systems. Computers chem. Engng., 20, S551-S556.

D. Ruppen, D. Bonvin and D.W.T. Rippin, (1997). Implementation of adaptive optimal operation for a semi-batch reaction system. Computers chem. Engng., to appear. 